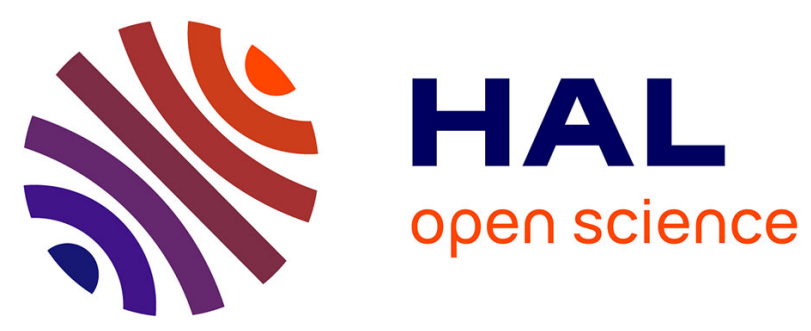

\title{
Four disulfide-bridged scorpion beta neurotoxin CssII: heterologous expression and proper folding in vitro.
}

Georgina Estrada, Blanca I. Garcia, Emanuele Schiavon, Ernesto Ortiz, Sandrine Cestèle, Enzo Wanke, Lourival D. Possani, Gerardo Corzo

\section{- To cite this version:}

Georgina Estrada, Blanca I. Garcia, Emanuele Schiavon, Ernesto Ortiz, Sandrine Cestèle, et al.. Four disulfide-bridged scorpion beta neurotoxin CssII: heterologous expression and proper folding in vitro.. Biochimica et Biophysica Acta - Molecular Cell Research, 2007, 1770 (8), pp.1161-8. 10.1016/j.bbagen.2007.04.006 . inserm-00378025

\section{HAL Id: inserm-00378025 https://www.hal.inserm.fr/inserm-00378025}

Submitted on 18 Jun 2009

HAL is a multi-disciplinary open access archive for the deposit and dissemination of scientific research documents, whether they are published or not. The documents may come from teaching and research institutions in France or abroad, or from public or private research centers.
L'archive ouverte pluridisciplinaire HAL, est destinée au dépôt et à la diffusion de documents scientifiques de niveau recherche, publiés ou non, émanant des établissements d'enseignement et de recherche français ou étrangers, des laboratoires publics ou privés. 


\section{Four disulfide-bridged scorpion beta neurotoxin CssII: heterologous expression and proper folding in vitro}

Georgina Estrada ${ }^{1}$, Blanca I. Garcia ${ }^{1}$, Emanuele Schiavon ${ }^{2}$, Ernesto Ortiz ${ }^{1}$, Sandrine Cestele $^{3}$, Enzo Wanke ${ }^{2}$, Lourival D. Possani ${ }^{1}$, and Gerardo Corzo ${ }^{1, *}$

${ }^{1}$ Departamento de Medicina Molecular y Bioprocesos, Instituto de Biotecnología, Universidad Nacional Autónoma de México, UNAM. Apartado Postal 510-3, Cuernavaca Morelos, 61500, MEXICO.

${ }^{2}$ Dipartimento di Biotecnologie e Bioscienze, Università di Milano-Bicocca, MILANO, 20126, Italy.

${ }^{3}$ Institut des Neurosciences Grenoble-GIN, IRTSV/CCFP-CEA GRENOBLE, 38054

Grenoble Cedex 09, France.

${ }^{*}$ To whom correspondence should be addressed: Instituto de Biotecnología - UNAM, Av. Universidad 2001, Cuernavaca, Morelos, 62210, Mexico. Tel. +52-777-317-1209; FAX +52-777-317-2388; Email: corzo@ibt.unam.mx 


\begin{abstract}
The gene of the four disulfide-bridged Centruroides suffusus suffusus toxin II was cloned into the expression vector pQE30 containing a 6His-tag and an FXa proteolytic cleavage region. This recombinant vector was transfected into E. coli BL21 cells and expressed under induction with isopropyl thiogalactoside (IPTG). The level of expression was $24.6 \mathrm{mg} / \mathrm{L}$ of culture medium, and the His tagged recombinant toxin (HisrCssII) was found exclusively in inclusion bodies. After solubilization the HisrCssII peptide was purified by affinity and hydrophobic interaction chromatography. The reverse-phase HPLC profile of the HisrCssII product obtained from the affinity chromatography step showed several peptide fractions having the same molecular mass of 9,392.6 $\mathrm{Da}$, indicating that HisrCssII was oxidized forming several distinct disulfide bridge arrangements. The multiple forms of HisrCssII after reduction eluted from the column as a single protein component of 9,400.6 Da. Similarly, an in vitro folding of the reduced HisrCssII generated a single oxidized component of HisrCssII, which was cleaved by the proteolytic enzyme FXa to the recombinant CssII (rCssII). The molecular mass of rCssII was 7,538.6 Da as expected. Since native CssII is amidated at the C-terminal residue whereas the rCssII is heterologously expressed in the format of free carboxyl end, there is a difference of 1Da, when comparing both peptides (native versus heterologously expressed). Nevertheless, they show similar toxicity when injected intracranially into mice, and both nCssII and rCssII show the typical electrophysiological properties of beta-toxins in $\mathrm{Na}_{\mathrm{v}} 1.6$ channels, which is for the first time demonstrated here. Binding and displacement experiments conducted with radiolabelled CssII confirms the electrophysiological results. Several problems associated with the heterologously expressed toxins containing four disulfide bridges are discussed.
\end{abstract}

Keywords: Centruroides suffusus suffusus, expression, protein folding, recombinant, scorpion, toxin

\title{
1. Introduction
}


Scorpion toxins that affect the voltage-gated sodium channels are essential tools for the study of their functional mechanism of action, for discrimination of different channel sub-types as well as for studying the structure-function relationship between channels and toxins. The sting of the buthid scorpion Centruroides suffusus suffusus is one of the most poisonous to the rural human population in the Northwest of Mexico, particularly to children, and it is a serious problem of Public Health in this region of the country, meanly in the State of Duranto [1]. CssII is the most abundant and noxious molecule of this venom, which is toxic to mammals and it is the principal component responsible for the toxicity of the whole venom in humans (unpublished results). The primary structure of CssII comprises 66 residues and 8 half-cystines that make four disulfide bridges. CssII binds with nanomolar affinity to the site-4 of the voltage-gated sodium channels [2].

Several communications have reported the production of recombinant voltagegated sodium channel toxins by heterologously expression in bacteria [3-9] and yeast [10]. However, in general these publications, even when expressing the same neurotoxin, do not present a clear description of their purification methods $[3,4,8,10]$, and most important yet, they have not included the demonstration of biological activity in vivo of their products $[3-5,8,10]$. One of the most important issues for heterelogous expression of proteins is to find the correct folding conditions, especially for correct disulfide bridge formation that allow the recombinant molecules to be biologically active. The number of possible structural forms for a protein rich in cysteines increases with the number of disulfide bridges in the molecule; consequently, to obtain a structural long-standing and in vivo functional recombinant protein with $n$ number of half-cystines, the right structure has to be one of the $\mathrm{N}$ ! / PD! $\mathrm{A}^{\mathrm{PD}}$ structural forms, where $\mathrm{N}$ is the number of half-cystines, $\mathrm{PD}$ is the number of cystines, and $\mathrm{A}$ is 2 (a disulfide arrangement). Therefore, the number of possible structural forms for CssII, a four disulfide-bridged scorpion toxin, which modifies sodium channel currents, is 105 . Unfortunately, some reports that have claimed to express successfully four disulfide-bridged neurotoxins have not demonstrated the correct folding and the biological activity of the product $[3-5,8,10]$. However, there are some reports (e.g. $[6,7,11-13])$ that succeeded to express a correct and folded toxin, by reduction and refolding the product in vitro; however, such toxins have from one to four extra residues attached to their N-terminal region. The interest of our research group is to obtain the 
expression of a voltage-gated sodium channel toxin with four disulfide-bridges, capable of reproducing the same structure and function (symptoms of intoxication) in animal models, as the native peptide does. Here we report a robust method for obtaining a recombinant CssII with similar secondary structure and biological activity in mice, as that of the native CssII. The electrophysiological characterization of both the native and the recombinant toxin is described. As it is demonstrated here for the first time that CssII is capable of modifying the gating mechanism of the sub-type of sodium channel $\left(\mathrm{Na}_{\mathrm{v}} 1.6\right)$.

\section{Material and methods}

\subsection{Bacterial strains, enzymes and plasmids.}

E. coli DH5- $\alpha$ was used for plasmid propagation. BL21 was used for the expression of the toxin-fusion proteins. Plasmids pKS (Stratagene, Amsterdam, The Netherlands) and pQE30 (Qiagen) were used for cloning and production of the fusion proteins with a 6His-tag, respectively. Restriction enzymes, Taq polymerase, Factor Xa and T4 DNA ligase were purchased from New England Biolabs.

\section{2. cDNA library construction}

A cDNA library was constructed with RNA extracted from a single telson (last postabdominal segment of the animal, which contains the stinger and a pair of venomous glands) of a C. suffusus suffusus scorpion. For RNA isolation the "Total RNA Isolation System" of Promega (Madison, WI) was used. With this material a full-length cDNA phagemid library was prepared using the SMART cDNA Library Construction Kit (CLONTECH Lab., Palo Alto, CA). The titer of the amplified cDNA library contained 1 x $10^{9}$ recombinant clones.

\subsection{Gene Cloning}

Based on the information obtained from direct peptide sequencing of CssII [2], an specific oligonucleotide was designed and used for the PCR reaction using as a template the cDNA material from the Library. The PCR reaction was performed in 1X Vent DNA polymerase buffer, $200 \mu \mathrm{M}$ dNTPs, $0.25 \mu \mathrm{M}$ forward primer (5'-ATA AAG AGG GCT 
ATC TGG-3') $0.25 \mu \mathrm{M}$; reverse CDS3'primer (5'-ATT CTA GAG GCC GAG GCG GCC GAC ATG-3') and 2 units of Vent DNA polymerase in a final volume of $50 \mu 1$ in a Perkin Elmer 9600 instrument. The reaction was incubated at $94^{\circ} \mathrm{C}$ for 5 min and $50{ }^{\circ} \mathrm{C}$ for $7 \mathrm{~min}$ before Vent polymerase was added. The mixture was then incubated at $72{ }^{\circ} \mathrm{C}$ for $1 \mathrm{~min}$ for one cycle. After the initial cycle, the mixture was incubated at $94^{\circ} \mathrm{C}$ for 30 $\mathrm{s}, 50^{\circ} \mathrm{C}$ for $40 \mathrm{~s}$ and $72^{\circ} \mathrm{C}$ for $30 \mathrm{~s}$ per 35 cycles, followed by a final $7 \mathrm{~min}$ step at $72^{\circ} \mathrm{C}$. PCR products were purified using a Centricon 100 column (Amicon) following the manufacturer instructions, and then ligated into a $p K S+E c o R V$ digested plasmid. The ligation reaction was used to transform competent E. coli DH5- $\alpha$ cells. Positive clones were sequenced from both ends using the Thermo sequenase radiolabeled terminator cycle sequencing kit (Amersham).

\subsection{Plasmid construction}

The DNA fragment encoding the CssII sequence, preceded by Factor Xa recognition site was amplified by PCR from a cDNA clone obtained from the library previously described (Figure 1). Thus, the plasmid contained: the 6His-tag, the sequence coding for the amino acids recognized by the protease (Factor Xa) and the gene of CssII. This construction was named pQE30XaCssII, and its product is here abbreviated: HisrCssII. It is also necessary to mention that we have corrected our initial clone found, for preferential codon usage according to E. coli. Also our first cloned gene had the amino acid glycine in position 27 (Gly27). The actually reported sequence of CssII contains Arg27. This substitution was also introduced in our pQE30XaCssII, in order to obtain the exact same amino acid sequence as originally described for CssII [2].

Appropriate oligonucleotides were designed for introducing the enzymatic restriction sites BamHI and PstI for directional insertion into pQE-30. The PCRamplified product was cloned into pKS, digested with EcoRV and subcloned using standard methods. The PCR amplifications were carried using the following oligonucleotides: CssIIB corresponds to GGATCCATCGAGGGAAGGAAAGAGGGCTATCTGGTAA-3'， and CssIIP: 5'CTGCAGTtACTAGTtGCATGTTTTATTAGGAAGG-3'. The PCR conditions were: 5 min at $94^{\circ} \mathrm{C}, 30$ cycles for $30 \mathrm{~s}$ at $94^{\circ} \mathrm{C}, 30 \mathrm{~s}$ at $55^{\circ} \mathrm{C}, 30 \mathrm{~s}$ at $72^{\circ} \mathrm{C}$, and a final extension 
of $5 \mathrm{~min}$ at $72^{\circ} \mathrm{C}$. The PCR products and the corresponding plasmids were digested with the corresponding restriction enzymes at $37^{\circ} \mathrm{C}$ overnight and purified by column (QIAquick) before ligation. The ligation reaction $(20 \mu 1)$ was carried out with T4 DNA ligase with a 10 folds insert excess over plasmid for $16 \mathrm{~h}$ at $16^{\circ} \mathrm{C}$. Ten microliters of the ligation reaction were used to transfect competent E. coli DH5- $\alpha$ cells. Positive clones with the expected insert were grown in LB ampicillin medium. The plasmids of positive colonies were purified by means of the High Pure Plasmid Isolation Kit (Roche). Plasmid constructs were verified by sequencing from both sites, the insert boundaries to confirm the reading frame and conservation of restriction sites. BL21 strains were transfected with the corresponding plasmid during $2 \mathrm{~min}$ at $42^{\circ} \mathrm{C}$, followed by $5 \mathrm{~min}$ in ice and $30 \mathrm{~min}$ recovery at $37^{\circ} \mathrm{C}$ in LB medium. Plates of LB contained $100 \mu \mathrm{g}$ of ampicillin.

\subsection{Overproduction and purification of HisrCssII.}

E. coli strain BL21 cells expressing the plasmid pQE30XaCssII were grown in LB medium. After the absorbance at $600 \mathrm{~nm}$ reached 0.5 of absorption units, the cultures were induced with $1 \mathrm{mM}$ IPTG (isopropyl- $\beta$-D-thiogalactopyranoside) for $6 \mathrm{~h}$ at $21{ }^{\circ} \mathrm{C}$. Cells were harvested by centrifugation (4000 x $g$ for $20 \mathrm{~min}$ ) using a Beckman centrifuges model J2-21, recovered in washing buffer (20 mM Tris-HCl, $\mathrm{pH} \mathrm{8.0)}$ and lysed with a French pressurizing device (900 psi). This material was centrifuged again (4000 x g for $20 \mathrm{~min}$ ). The insoluble fraction was resuspended twice with the washing buffer and centrifuged in the same conditions. The recombinant toxin was extracted from the inclusion bodies using $6 \mathrm{M}$ guanidinium chloride $(\mathrm{GndHCl})$ in a $0.05 \mathrm{M}$ Tris-base, buffer, $\mathrm{pH} 8.0$ and centrifuged for $40 \mathrm{~min}$ in a refrigerated Hettich Universal 32R centrifuge. The supernatant was purified by affinity column. Purification of the HisrCssII by Ni-NTA (Ni-nitrilotriacetic acid) affinity column chromatography was performed according to the instructions of the manufacturer (Qiagen) using denaturing conditions with buffer $\mathrm{A}(6 \mathrm{M}$ GndHCl in a $0.05 \mathrm{M}$ Tris-base buffer, $\mathrm{pH} 8.0)$ and buffer $\mathrm{B}(6 \mathrm{M}$ GndHCl in $0.05 \mathrm{M}$ Tris-base buffer, containing $400 \mathrm{mM}$ imidazole, $\mathrm{pH}$ 8.0). Buffer B was eliminated by a second purification step under reverse-phase HPLC. An analytic $\mathrm{C}_{18}$ reverse-phase column (Nacalai-Tesque Japan) was run from solvent A $(0.1 \%$ trifluoroacetic acid - TFA- in water) to solvent B (0.1\% TFA in acetonitrile). The HPLC 
system previously described [14] was used for this separation, and the gradient was run from $20 \%$ to $60 \%$ solvent B, during $40 \mathrm{~min}$. The HisrCssII product was vacuum dried and reduced using $50 \mathrm{mM}$ DTT in $0.05 \mathrm{M}$ Tris-base buffer, $\mathrm{pH}$ 8.0. The recombinant product was allowed to fold under controlled conditions using $2 \mathrm{M} \mathrm{GndHCl}$ in $0.05 \mathrm{M}$ Tris-base buffer, $\mathrm{pH} 8.0$, containing $1 \mathrm{mM}$ reduced glutathione $(\mathrm{GSH}) / 0.1 \mathrm{mM}$ oxidized glutathione (GSSG). Recombinant HisrCssII was cleaved by FXa using a ratio 50:1 respectively in $10 \mathrm{mM}$ Tris-base buffer, $\mathrm{pH} 8.0$, at room temperature for $14 \mathrm{~h}$. The full recombinant CssII (rCssII) was cleaved from the His-tail by FXa enzymatic treatment and eluted from a reverse-phase column, vacuum dried and stored at $-20^{\circ} \mathrm{C}$. The identity of the toxin was confirmed by both automatic Edman degradation and mass spectrometry analysis using a Finnigan $\mathrm{LCQ}^{\mathrm{DUO}}$ ion trap mass spectrometer (San Jose, CA, USA). The two last techniques used for chemical identification of proteins are currently being used in the laboratory and are well documented elsewhere [14].

\subsection{Gel electrophoresis}

The resolving and stacking gel solutions are modified versions of the Laemmli system [15]. The $12.5 \%$ resolving gel solution $(6.5 \mathrm{ml})$ containing $375 \mathrm{mM}$ Tris $(\mathrm{pH}$ 8.6), $0.1 \%$ SDS, $12.5 \%$ acrylamide/bisacrylamide solution (38:1), 0.05\% APS, and $0.05 \%$ TEMED was well mixed in that order. The $2 \%$ stacking gel solution (containing $375 \mathrm{mM}$ Tris (pH 6.8), 0.1\% SDS, 2\% acrylamide/bisacrylamide solution (38:1), 0.05\% APS, and $0.1 \%$ TEMED) was added on top of the resolving gel. The comb was inserted, and the stacking gel was allowed to stand until polymerization (approximately $1 \mathrm{~h}$ ).

The sample protein concentration was measured and adjusted to $6 \mathrm{mg} / \mathrm{ml}$ with lyses buffer. An equal volume of $2 \mu 1$ SDS sample buffer was added, and the mixture was heated at $100{ }^{\circ} \mathrm{C}$ for $3 \mathrm{~min}$. The electrode chamber was filled with the running buffer (25 $\mathrm{mM}$ Tris, $192 \mathrm{mM}$ glycine, and 0.1\% SDS, $\mathrm{pH}$ 8.3), and samples $(12 \mu \mathrm{g}$ proteins/lane) were subjected to analysis. Unstained high-molecular weight protein standard served as a reference band to ensure accurate molecular weight identification. Gel electrophoresis was performed at a constant current of $6.5 \mathrm{~mA} /$ plate for $2 \mathrm{~h}$ at room temperature and at a constant $100 \mathrm{~V}$ in succession until the bromophenol blue reached approximately $5 \mathrm{~mm}$ above the bottom of the gel. After electrophoresis, the gel slabs 
were stained with Coomassie brilliant blue R-250 in 25\% isopropanol and $10 \%$ acetic acid and subsequently were destained in a 10\% methanol and 5\% acetic acid solution.

\subsection{Circular Dichroism (CD) measurements}

CD spectra were obtained on a Jasco J-725 spectropolarimeter (Jasco, Japan). The spectra were measured from 260 to $189 \mathrm{~nm}$ in $60 \%$ trifluoroethanol to promote hydrogenbonding [16], $\mathrm{pH} 7.1$ at room temperature, with a $1 \mathrm{~mm}$ path-length cell. Data were collected at $0.1 \mathrm{~nm}$ with a scan rate of $50 \mathrm{~nm} / \mathrm{min}$ and a time constant of $0.5 \mathrm{~s}$. The concentration of the toxins rCssII, native CssII and HisrCssII was $250 \mu \mathrm{g} / \mathrm{ml}$. Data were the average of three separate recordings and analyzed according to Andrade et al. [17].

\subsection{Biological activity}

The protocol used for assaying the activity of these peptides in vivo, using the mice model, was followed according to the guidelines of our Institute Committee of Animal Welfare, keeping the number of animals to a minimum required to validate the experiments.

Male mice (CD-1, 20 g body weight) were tested by intracranial and intra-peritoneal injection. Pure peptides HisrCssII, rCssII, and CssII were diluted up to $5 \mu$ (for intracranial inoculation) or $200 \mu \mathrm{l}$ (intra-peritoneal inoculation) with bovine serum albumin (BSA) solution $(20 \mathrm{mg} / \mathrm{ml}$ in $0.9 \% \mathrm{NaCl})$ and injected with a $10 \mu \mathrm{l}$ microsyringe fitted with a glass capillary. The injection was performed mid-way between the left eye and the left ear (intracranial) or with $1 \mathrm{ml}$ syringe into the peritoneal cavity (intraperitoneal), respectively. Negative controls were done with saline solution only and positive controls with the neurotoxic scorpion peptide LqhIV isolated in our laboratory [18]. Mice were observed for toxicity symptoms up to 24 hours.

\subsection{Electrophysiological experiments}

2.9.1 Cell culture. The HEK293 cell line stably expressing human $\mathrm{Na}_{\mathrm{v}} 1.6$ (generously donated by Dr. J.J. Clare of GlaxoSmithKline, Medicines Res. Centre, Gunnels Wood 
Rd., Stevenage, Herts SG1 2NY, UK) was cultured in modified Dulbecco's medium supplemented with $10 \%$ fetal bovine serum as described by [19].

2.9.2 Solutions and drugs. The standard extracellular solution contained (mM): $\mathrm{NaCl}$ 130, $\mathrm{KCl} 5, \mathrm{CaCl}_{2} 2, \mathrm{MgCl}_{2}$ 2, HEPES-NaOH 10, D-glucose 5, $\mathrm{pH}$ 7.40. The standard pipette solution contained (mM): $\mathrm{K}^{+}$-aspartate $130, \mathrm{NaCl} 10, \mathrm{MgCl}_{2}$ 2, EGTA-KOH 10, Hepes- $\mathrm{KOH}$ 10, $\mathrm{pH}$ 7.3. Known quantities of the toxins were dissolved in the extracellular solution immediately before the experiments. Tetrodotoxin (TTX, Sigma, Italy) was used for correction purposes at $100 \mathrm{nM}$.

2.9.3 Patch-clamp recordings and data analysis. The extracellular solutions were delivered through a remote-controlled 9-hole $(0.6 \mathrm{~mm})$ linear positioner placed near the cell under study; the average response time was 2-3 seconds. The currents were recorded at room temperature using the MultiClamp 700A (Axon Instruments, USA) as previously described $[19,20]$. In brief, pipette resistance was about 1.3-2.1 MOhms; cell capacitance and series resistance errors were carefully (85-90\%) compensated for, before each run of the voltage clamp protocol in order to reduce voltage errors to less than $5 \%$ of the protocol pulse. The $\mathrm{P} / \mathrm{N}$ leak procedure, pClamp 8.2 (Axon Instruments, USA) and Origin 7 (Microcal Inc., USA) software were routinely used during data acquisition and analysis.

\subsection{Radio-iodination experiments}

2.10.1 Neuronal membrane preparation. Rat brain synaptosomes were prepared from adult albino Wistar rats. Homogenization of rat brain was performed in $0.32 \mathrm{M}$ sucrose buffer containing $20 \mathrm{mM}$ HEPES-Tris, $\mathrm{pH}$ 7.4. Following centrifugation at 1,000 $\mathrm{g}$ for 5 minutes, the supernatant was recentrifuged at $10,000 \mathrm{~g}$ for $20 \mathrm{~min}$ (P2 fraction). The pellet was resuspended in the buffer used for the homogenization. 
2.10.2 Radioiodination. CssII was radio-iodinated by lactoperoxydase as previously described [21] using $1 \mathrm{nmol}$ of toxin and $1 \mathrm{mCi}$ of carrier-free $\mathrm{Na}^{125} \mathrm{I}$. The monoiodotoxin was purified as described previously [22].

2.10.3 Binding assays. Equilibrium competition assays were performed using increasing concentrations of unlabelled toxin in the presence of a constant low concentration of the radioactive toxin. The binding medium composition was; Choline-Cl, $130 \mathrm{mM}$; $\mathrm{KCl}, 5.4$ $\mathrm{mM}$; Glucose, $5.5 \mathrm{mM}$; HEPES $50 \mathrm{mM}$ and BSA $2 \mathrm{mg} / \mathrm{ml}, \mathrm{pH}$ 6.5. Rat brain synaptosomes were suspended in $0.2 \mathrm{ml}$ binding buffer containing ${ }^{125}$ I-CssII. After incubation $1 \mathrm{~h}$ at room temperature, the reaction mixture was diluted with $3 \mathrm{ml}$ ice-cold wash buffer and filtered through $\mathrm{GF} / \mathrm{C}$ filters under vacuum. Filters were rapidly washed twice with additional $3 \mathrm{ml}$ buffer. Non-specific binding was determined in the presence of $70 \mathrm{nM}$ unlabelled CssII and consist typically of 5-9\% of the total binding. Each experiment was performed at least three times.

\section{Results and Discussion}

\subsection{Gene cloning and recombinant expression}

As described in the Material and Methods section, a gene coding a CssII-like toxin was found (Fig. 1A). However, its sequence analysis revealed a discrepancy in the DNA with respect to the amino acid sequence determined by Edman degradation using the native toxin. In position 27 the cDNA showed a Gly substituting for an Arg [2]. Also it is noteworthy to mention that the native CssII is aminated at its C-terminus (Fig. $1 \mathrm{~A}$ ). Moreover, the Leu26 codon in the DNA sequence corresponded to a low usage codon in E. coli, so we decided to change it for another codon of Leu different from that found. In order to have a nucleotide sequence that would code for the same native toxin, appropriate oligonucleotides were synthesized and used for re-amplification of the mutagenized DNA introducing the vector-compatible restriction sites. The mutagenic oligonucleotide was CssIIG27R: 5'-CTG TTT GCA TTC GCG CAG GCA ATA ATC GTT-3' (30-mer, the mutagenic triplets are showed underlined), and the amplification primers were CssIIB: 5'-GCG GAT CCA TCG AGG GAA GGA AAG AAG GCT ATC 
TGG TAA G-3' (40-mer, the HindIII site is showed underlined) and CssIIP: 5'-AAA ACT GCA GTT ACT AGT TGC ATG TTT TAT TAG GAA GG-3' (38-mer, the Pst I site is showed underlined). The amplification was performed in two steps. The first (mutagenic) PCR employed oligos CssIIB and CssIIG27R, (8 cycles, using $50^{\circ} \mathrm{C}$ as annealing temp., followed by 25 cycles with $55^{\circ} \mathrm{C}$ as annealing temperature). The purified product of the first PCR was then used as mega-primer for the second (amplification) PCR together with oligo CssIIP $\left(8\right.$ cycles, using $50^{\circ} \mathrm{C}$ as annealing temperature), followed by 25 cycles with $60^{\circ} \mathrm{C}$ as annealing temperature). The product with the expected size ( $230 \mathrm{bp}$ ) was purified with the QIAquick Gel Extraction Kit (QIAGEN), blunt-end cloned into EcoRV-digested pBluescriptKS+ vector (Stratagene) and its sequence was verified. As indicated in Material and Methods, the plasmid pQ30XaCssII was constructed introducing extra amino acid sequences in the gene for the improving purification and proper excision of the mature rCssII (Fig. 1B-C). Therefore, this plasmid contained the information to code a segment of six histidines followed by four amino acids corresponding to cleavage site of the enzyme Factor Xa, and the 66 amino acid residues corresponding to the mature toxin II isolated from C. suffusus suffusus.

E. coli strain BL21 cells expressed the His tagged FXa recombinant CssII peptide as shown in Fig. 2. Lane 3 of this figure clearly shows the presence of an intense protein band with the molecular weight expected for HisrCssII. It is important to mention that this band was absent in SDS-PAGE-gel corresponding to the soluble extract (data not shown). Hence, it seems that the entire recombinant product expected goes to the inclusion bodies.

\subsection{Purification, in vitro folding and enzymatic cleavage}

The cells obtained after induction with IPTG and decanted by centrifugation were resuspended and ruptured with the French Press, as described in Material and Methods. After solubilization with $\mathrm{GndHCl}$ the supernatant was purified by affinity column (see same section), and the imidazol eluate was directly loaded into the $\mathrm{C}_{18}$ column for HPLC separation. The results of this chromatogram are shown in Fig. 3 (label A, multiple oxidized forms). Interestingly, all the chromatographic peaks labeled from 1 to 10 had the 
expected molecular mass of the recombinant HisrCssII peptide (9,392.6 Da) and showed symptoms of intoxication when injected intracraneally into mice ( $20 \mu \mathrm{g}$ protein each), except fractions 1, 2, 9 and 10. Fractions 4-6 were lethal at this dose. This fact suggests that very likely fractions 4-6 were folded in the correct way, or close enough to sustain the biological effect. The idea that isoforms could have been generated during oxidation of the thiol groups for formation of the disulfide bridges prompted us to conduct an in vitro reduction-oxidation step. Fig. 3 (label B, reduced HisrCssII) shows the results of the HPLC separation after chemical reduction of a pool of fractions with DTT. In this graphic, we have superimposed both the results of the HPLC separation of multiple oxidized forms (Fig. 3 label A) and the single reduced form (Fig. 3 label B) in order to show the transformation of the several sub-fractions into only one: the reduced form that elutes latter in this chromatogram. Therefore, a single fraction with a molecular mass of 9,400.6 Da containing $8 \mathrm{Da}$ more than the expected mass because of the reduction of 4 disulfide bridges was found confirming that HisrCssII was oxidized in multiple forms inside the E. coli cell or during our extraction procedure. The reduced HisrCssII was folded in vitro conditions yielding a single oxidized fraction (Fig. 4A label A). The molecular mass obtained was the expected one $(9,392.6 \mathrm{Da})$ for a fully folded molecule. In this graphic, we have again superimposed both the results of the HPLC separation of the reduced form (Fig. 4A label B) and the single oxidized form (Fig. 4A label A) to show the transformation of the reduced form. The in vitro folded HisrCssII from Fig. 4A (label A) was cleaved with the endoprotease FXa (see Materials and Methods). After enzymatic treatment, two fractions with the molecular mass of 9,392.6 and 7,538.6 $\mathrm{Da}$ were obtained (Fig. 4B). The molecular mass of the first fraction represents the uncleaved HisrCssII protein and the second one was the rCssII. The Edman degradation of the rCssII peptide confirmed that the first 5 amino acid residues correspond to the expected sequence of a bona fide toxin CssII.

In a typical experiment, starting with $1 \mathrm{~L}$ of cell culture, the amount of recombinant HisrCssII obtained was $24.6 \mathrm{mg}$ protein. After the DTT reduction $(16.4 \mathrm{mg})$, in vitro folding $(5.6 \mathrm{mg})$, cleavage with factor Xa and the final HPLC purification step, the amount of rCssII obtained was $2.6 \mathrm{mg} / \mathrm{L}$. The in vitro folding of CssII was $34.1 \%$. This yield of renaturation is substantial compared to other higher molecular mass proteins 
obtained by recombinant DNA [23]. However, our experience in synthesizing short peptides and folding them is that according to the primary structure we could obtain from 0 to $45 \%$ renaturation [24]. Concerning recombinant scorpion toxins, as an example, Zilberberg et al. [6] renatured the recombinant LqhoIT insect toxin with a yield of active toxin of $5-10 \mathrm{mg} / \mathrm{L}$ from $50 \mathrm{mg} / \mathrm{L} \mathrm{E}$. coli inclusion bodies; that is, a renaturation yield of $20-40 \%$. The same research team obtained $1.2 \%$ yield (after denaturation and renaturation) of the recombinant toxin LqhIT2 [7]. Similarly, renaturation of toxin Aah1 performed by M'Barek et al [25] showed a recovery yield that varies from $0.3 \%$ to $2 \%$

efficiency after refolding, whereas expression and renaturation of the excitatory insectspecific toxin BmK IT-AP from Buthus martensii showed $0.5 \mathrm{mg} / \mathrm{L}$ of culture [5]. Some toxins just fold nicely, but unfortunately others do not. The primary sequence of betatoxins CssII (this work), BmK IT-AP [5], CssIV [13] and Bj-rIT [12] are examples of toxins that are easily refolded in vitro with good yields (30-40\%).

\subsection{Evidence of similar chemical and structural characteristics}

Although the C-terminal amino acids of native CssII and rCssII are different, both CssII and rCssII had similar elution times under similar reverse-phase HPLC conditions (data not shown). The native CssII has an amidated C-terminus, and rCssII has a carboxylated C-terminus. Nevertheless, the circular dichroism of CssII, rCssII and HisrCssII (Fig. 5) showed low absorption for the $\alpha$-helix secondary structure and a high content in $\beta$-sheets similar to the structure of most known $\mathrm{Na}^{+}$channel scorpion toxins $[26,27]$. The three peptides adopt the typical $\alpha / \beta$ motif of most $\mathrm{Na}^{+}$channel scorpion toxins that are characterized by negative ellipticities at 208 and $222 \mathrm{~nm}$, and positive bands at $196 \mathrm{~nm}[26,27]$. Similar secondary structure fractions using aqueous TFE have been previously observed in $\mathrm{Na}^{+}$channel scorpion toxins containing $\alpha / \beta$ structures $[18$, 27].

\subsection{Biological activity}

The $\mathrm{LD}_{50}$ of the native CssII has been reported to be $5 \mathrm{ng} / 20 \mathrm{~g}$ mice when injected intracranially [2]. Therefore groups of 5 mice each were injected with the equivalent of 3 $\mathrm{LD}_{50}$ of the native toxin; that is $15 \mathrm{ng} / 20 \mathrm{~g}$ mice of CssII, rCssII or HisrCssII, 
respectively. The value of $3 \mathrm{LD}_{50}$ was chosen because a dose-response follows a Normal Cumulative Distribution Function, and this dose represents the probability of $\mathrm{p}>0.995$, under the dose-response curve. It means a $>99.5 \%$ confidence that $3 \mathrm{LD}_{50}(15 \mathrm{ng} / 20 \mathrm{~g}$ mice) will kill all animals tested if rCssII and HisrCssII have similar toxicity as the native CssII. The value of $3 \mathrm{LD}_{50}$ is an approximation to the $\mathrm{LD}_{100}$. The groups of mice that were injected with CssII and rCssII did not survive the injection of 3 LD $_{50}$ indicating that CssII and rCssII have similar toxicity at the concentration used. Only the group of mice that was injected with HisrCssII survived to this treatment indicating that HisrCssII has lower activity than that of CssII and rCssII. HisrCssII was lethal to mice only at $15 \mathrm{LD}_{50}$ (75 ng/mice). When mice were injected intraperitoneally, the $\mathrm{LD}_{100}$ for CssII, rCssII and HisrCssII was observed at 3,6 and $15 \mu \mathrm{g} / 20 \mathrm{~g}$ mice, respectively.

To investigate the specific functional role of these peptides we performed also specific electrophysiological experiments to assess the activity of nCssII and rCssII on cells expressing the $\mathrm{Na}_{\mathrm{v}} 1.6$ isoform of the voltage-gated sodium channels. The typical $\beta$ scorpion toxin left-shift of the normalized conductance versus membrane potential is shown in Fig. 6A and 6B for the native and recombinant peptides, respectively. Data (see legend) indicate that a fraction of the channels change their biophysical properties according to known properties which have been already studied before by using others $\beta$ scorpion toxins, such as CssIV of this species and $\mathrm{Cn} 2$ from Centruroides noxius venom [20]. It is worth mentioning that the three toxins mentioned here were shown to affect the same sub-type of Na-channel, although with some particular differences concerning the resurgent currents (see reference [20]).

Similarly to the in vivo experiments, nCssII had more pronounced effects on the $\mathrm{Na}_{\mathrm{v}} 1.6$ channels than that of rCssII, at the same concentration (Fig. 6A and 6B). Competitive binding experiments support the electrophysiological data. As shown in Fig. $6 \mathrm{C}$, the native CssII inhibits ${ }^{125} \mathrm{I}$-CssII binding with an $\mathrm{IC}_{50}$ of $0.10 \mathrm{nM}$. On the other hand, the $\mathrm{IC}_{50}$ observed for rCssII was $1.5 \mathrm{nM}$. Although the $\mathrm{IC}_{50}$ of the $\mathrm{rCssII}$ is 15 times less efficient than the native toxin, the fact that it binds at a nanomolar concentration to the synaptosomes is a good indication that the recombinant peptide is corrected folded. The difference found is certainly due to the difference of the C-terminal amino acid, which is not amidated in the case of the rCssII. Actually this goes in the same direction of 
earlier reported work by Lebrun et al. 1997 [28] found for toxin HsTx1 of Heterometrus spinnifer scorpion venom. The authors showed that lack of amidation of HsTx1 decreases the binding affinity to the synaptosomes membranes. On the whole, all these biological activity data not only converge in showing similar differences in the effects, but also suggest that probably these peptides will affect also other $\mathrm{Na}^{+}$channel isoforms that are expressed in brain [29]. Experiments are in progress to clarify this point. Meanwhile, the fact that CssII affects Nav1.6, which has been detected during developmental myelination and during remyelination at the node of Ranviers in the peripheral nervous system [30], correlates well with the observation that CssII cause most of the subcutaneous toxicity of the sting of C.s. suffusus.

The E. coli system used for the recombinant expression of rCssII is not capable of producing a $\mathrm{C}$-terminal asparagine amidated form as the specialized cells of the venom glands of the scorpion do, but the rCssII have the same in vivo activity. It has been observed in sodium channel neurotoxins from spiders and scorpions that C-terminus amidated peptides have stronger affinity for their sodium channel receptors [9, 24]; that is, the exchange of an anionic charge for a neutral one at the C-terminus increases the overall positive charge of amidated peptides which is correlated with their receptor affinities [31].

In our opinion, this communication reports in detail the misfolded products of a four-disulfide bridge toxin (rCssII) when expressed in a heterologous system. As it was mentioned in the Introductory section of this report, this is an important contribution due to the fact that most literature concerning this subject have failed to achieve a correct, fully folded toxin, when dealing with a small peptide heterologously expressed, which contains 8 cysteines and can theoretical form up to 105 isoforms, if considered only the disulfide pairing possibility. Obviously, in the scorpion, the molecular machinery in charge of its production directs the formation of the correct folding.

In previous publications a modified version of rCssII was reported, in a thioredoxin mutant strain of E. coli (see reference 3), but no attention was given to describe the possible multiple isoforms generated during heterologous expression, neither in which form the toxin was finally obtained. The unidimensional NMR data presented is not accompanied by a control experiment with native CssII. The biological assay included refers to inhibition of $\left[{ }^{3} \mathrm{H}\right] \mathrm{GABA}$ uptake in $\mathrm{PC} 12$ cells in vitro, but this method certainly 
lacks more precision for the purpose of showing exactly the same behavior as the native toxin. Direct lethality tests on animals were not performed neither fine electrophysiological assays are shown. At least two other publications concerning a successful heterogously expression of scorpion toxins containing four disulfide bridges were reported using toxins LqhoIT and LqhIT2 from the scorpion Leiurus quinquestriatus hebraeus [6, 7]. The product of expression was recovered from inclusion bodies, but no specification of

possible miss folded isoforms was discussed, although they did use denaturation and refolding in vitro, as we describe here. Additionally their recombinant toxins had an extra amino acid at the $\mathrm{N}$-terminal side. For these reasons, we are confident that our results, open the field for production of site-directed mutants to study the structure-function relationship of this toxin with voltage-dependent sodium channels (Nav1.6) as well as for research of antivenom production, using recombinant material.

\section{Acknowledgements}

This work was supported by grants from Institute Bioclón S.A., CONACyTMOR-2004-C02-002, and DGAPA IN226006 (UNAM) to L.D.P. and G.C. repectively, and MIUR-PRIN 2005-2001055320 to EW. The confirmation of the amino acid sequence of rCssII by Dr. Fernando Zamudio and the mass spectrometry determination conducted by Dr. Cesar Batista are greatly acknowledged. The authors also acknowledge the kind assistance of Timoteo Olamendi and advice of Blanca Ramos and Alejandro Olvera for cloning pQE30XaCssII, and Dr. Gloria Saab for CD spectra. ES is a postDoc at the University of Milano-Bicocca.

\section{References}

[1] L. Vega Franco, M. Lia Jaime, [Epidemiologic considerations on scorpion stings in Durango city], Rev Invest Salud Publica 26 (1966) 7-21.

[2] M. F. Martin, L. G. Garcia y Perez, M. el Ayeb, C. Kopeyan, G. Bechis, E. Jover, H. Rochat, Purification and chemical and biological characterizations of seven toxins from the Mexican scorpion, Centruroides suffusus suffusus, J. Biol. Chem. 262 (1987) 4452-4459. 
[3] T. M. Johnson, M. W. Quick, T. T. Sakai, N. R. Krishna, Expression of functional recombinant scorpion beta-neurotoxin Css II in E. coli, Peptides 21 (2000) 767772.

[4] C. Legros, B. Ceard, H. Vacher, P. Marchot, P. E. Bougis, M. F. MartinEauclaire, Expression of the standard scorpion alpha-toxin AaH II and AaH II mutants leading to the identification of some key bioactive elements, Biochim Biophys Acta 1723 (2005) 91-99.

[5] C. Li, W. Liu, F. Bossmans, R. H. Zhu, J. Tytgat, D. C. Wang, Expression, renaturation and functional analysis of an excitatory insect-specific toxin from scorpion Buthus martensii Karsch, Protein Pept Lett 12 (2005) 635-638.

[6] N. Zilberberg, D. Gordon, M. Pelhate, M. E. Adams, T. M. Norris, E. Zlotkin, M. Gurevitz, Functional expression and genetic alteration of an alpha scorpion neurotoxin, Biochemistry 35 (1996) 10215-10222.

[7] M. Turkov, S. Rashi, Z. Noam, D. Gordon, R. Ben Khalifa, M. Stankiewicz, M. Pelhate, M. Gurevitz, In vitro folding and functional analysis of an anti-insect selective scorpion depressant neurotoxin produced in E. coli, Protein Expr Purif 10 (1997) 123-131.

[8] S. Banerjee, E. V. Curto, M. Beckman, G. B. Brown, J. Zhong, N. R. Krishna, Expression of functional scorpion neurotoxin Lqq-V in E.coli, Peptides 27 (2006) 49-54.

[9] K. Benkhadir, R. Kharrat, S. Cestele, A. Mosbah, H. Rochat, M. El Ayeb, H. Karoui, Molecular cloning and functional expression of the alpha-scorpion toxin BotIII: pivotal role of the C-terminal region for its interaction with voltagedependent sodium channels, Peptides 25 (2004) 151-161.

[10] M. F. Martin-Eauclaire, M. Sogaard, C. Ramos, S. Cestele, P. E. Bougis, B. Svensson, Production of active, insect-specific scorpion neurotoxin in yeast, Eur J Biochem 223 (1994) 637-645.

[11] B. Bouhaouala-Zahar, R. Benkhalifa, N. Srairi, I. Zenouaki, C. Ligny-Lemaire, P. Drevet, F. Sampieri, M. Pelhate, M. El Ayeb, A. Menez, H. Karoui, F. Ducancel, A chimeric scorpion alpha-toxin displays de novo electrophysiological properties similar to those of alpha-like toxins, Eur J Biochem 269 (2002) 2831-2841. 
[12] L. Cohen, I. Karbat, N. Gilles, O. Froy, G. Corzo, R. Angelovici, D. Gordon, M. Gurevitz, Dissection of the functional surface of an anti-insect excitatory toxin illuminates a putative "hot spot" common to all scorpion beta-toxins affecting $\mathrm{Na}+$ channels, J. Biol. Chem. 279 (2004) 8206-8211.

[13] L. Cohen, I. Karbat, N. Gilles, N. Ilan, M. Benveniste, D. Gordon, M. Gurevitz, Common features in the functional surface of scorpion beta-toxins and elements that confer specificity for insect and mammalian voltage-gated sodium channels, $\mathrm{J}$ Biol Chem 280 (2005) 5045-5053.

[14] A. Prochnicka-Chalufour, G. Corzo, H. Satake, M. F. Martin-Eauclaire, A. R. Murgia, G. Prestipino, G. D'Suze, L. D. Possani, M. Delepierre, Solution structure of discrepin, a new $\mathrm{K}+$-channel blocking peptide from the alpha-KTx15 subfamily, Biochemistry 45 (2006) 1795-1804.

[15] U. K. Laemmli, Cleavage of structural proteins during the assembly of the head of bacteriophage T4, Nature 227 (1970) 680-685.

[16] F. D. Sonnichsen, Van Eyk, J.E., Hodges, R.S., Sykes, B.D., Effect of trifluoroethanol on protein secondary structure: an NMR and CD study using a synthetic actin peptide, Biochemistry 31 (1992) 8790-8798.

[17] M. A. Andrade, P. Chacón, J. J. Merelo, F. Morán, Evaluation of secondary structure of proteins from UV circular dichroism using an unsupervised learning neural network, Prot. Eng. 6 (1993) 383-390.

[18] G. Corzo, E. Villegas, T. Nakajima, Isolation and structural characterization of a peptide from the venom of scorpion with toxicity towards invertebrates and vertebrates, Prot. Pep. Lett. 8 (2001) 385-393.

[19] J. S. Oliveira, E. Redaelli, A. J. Zaharenko, R. Restano Cassulini, K. Konno, D. C. Pimenta, J. C. Freitas, J. J. Clare, E. Wanke, Binding specificity of sea anemone toxins to Nav 1.1-1.6 sodium channels: unexpected contributions from differences in the IV/S3-S4 outer loop, 279 (2004) 33323-33235.

[20] E. Schiavon, T. Sacco, R. Cassulini, G. Gurrola, F. Tempia, L. D. Possani, E. Wanke, Resurgent current and voltage sensor trapping enhanced activation by a beta-scorpion toxin solely in Nav1.6 channel. Significance in mice Purkinje neurons, J. Biol. Chem. 281 (2006) 20326-20337. 
[21] S. Cestele, Y. Qu, J. C. Rogers, H. Rochat, T. Scheuer, W. A. Catterall, Voltage sensor-trapping: enhanced activation of sodium channels by beta-scorpion toxin bound to the S3-S4 loop in domain II, Neuron 21 (1998) 919-931.

[22] S. Cestele, C. Kopeyan, R. Oughideni, P. Mansuelle, C. Granier, H. Rochat, Biochemical and pharmacological characterization of a depressant insect toxin from the venom of the scorpion Buthacus arenicola, Eur J Biochem 243 (1997) 93-99.

[23] K. E. Volynski, E. D. Nosyreva, Y. A. Ushkaryov, E. V. Grishin, Functional expression of alpha-latrotoxin in baculovirus system, FEBS Lett 442 (1999) 2528.

[24] G. Corzo, P. Escoubas, E. Villegas, I. Karbat, D. Gordon, M. Gurevitz, T. Nakajima, N. Gilles, A spider toxin that induces a typical effect of scorpion alphatoxins but competes with beta-toxins on binding to insect sodium channels, Biochemistry 44 (2005) 1542-1549.

[25] S. M'Barek, Z. Fajloun, S. Cestele, C. Devaux, P. Mansuelle, A. Mosbah, B. Jouirou, M. Mantegazza, J. Van Rietschoten, M. El Ayeb, H. Rochat, J. M. Sabatier, F. Sampieri, First chemical synthesis of a scorpion alpha-toxin affecting sodium channels: the Aah I toxin of Androctonus australis hector, J Pept Sci 10 (2004) 666-677.

[26] E. P. Loret, F. Sampieri, A. Roussel, C. Granier, H. Rochat, Conformational flexibility of a scorpion toxin active on mammals and insects: a circular dichroism study, Proteins 8 (1990) 164-172.

[27] E. P. Loret, M. F. Martin-Eauclaire, P. Mansuelle, F. Sampieri, C. Granier, H. Rochat, An anti-insect toxin purified from the scorpion Androctonus australis Hector also acts on the alpha- and beta-sites of the mammalian sodium channel: sequence and circular dichroism study, Biochemistry 30 (1991) 633-640.

[28] B. Lebrun, R. Romi-Lebrun, M. F. Martin-Eauclaire, A. Yasuda, M. Ishiguro, Y. Oyama, O. Pongs, T. Nakajima, A four-disulphide-bridged toxin, with high affinity towards voltage-gated $\mathrm{K}+$ channels, isolated from Heterometrus spinnifer (Scorpionidae) venom, Biochem J 328 ( Pt 1) (1997) 321-327. 
[29] J. H. Caldwell, K. L. Schaller, R. S. Lasher, E. Peles, S. R. Levinson, Sodium channel Nav1.6 is localized at nodes of Ranvier, dendrites, and synapses, Proc. Natl. Acad. Sci. USA 97 (2000) 5616-5620.

[30] D. P. Schafer, A. W. Custer, P. Shrager, M. N. Rasband, Early events in node of Ranvier formation during myelination and remyelination in the PNS, Neuron Glia Biol. 2 (2006) 69-79.

[31] G. Ferrat, F. Bosmans, J. Tytgat, C. Pimentel, B. Chagot, N. Gilles, T. Nakajima, H. Darbon, G. Corzo, Solution structure of two insect-specific spider toxins and their pharmacological interaction with the insect voltage-gated $\mathrm{Na}^{+}$channel, Proteins 59 (2005) 368-379. 


\section{Figures legend}

Fig. 1. CssII-like gene and construction of the plasmid carrying CssII.

A). The nucleotide and amino acid sequence of CssII-like. B). Scheme of the expression vector pQE30XaCssII. The His-tag is part of the 5'-nucleotide sequence of pQE30, in such way that the sequence to be expressed is the one shown, in which the cleavage site for the restriction enzyme are indicated (BamHI and PstI). C). The oligonucleotides used for PCR.

Fig. 2. SDS-PAGE showning the expression of HisrCssII.

Lane 1 shows the molecular weight markers. Lane 2 and 4 the profile of proteins after induction. Lane 3, protein pattern after affinity column (upper bands are oligomers of HisrCssII). The protein band corresponding to HisrCssII is indicated by arrow. Lane 5, the protein content without IPTG induction. The content of protein applied in each lane was approximately $5 \mu \mathrm{g}$.

Fig. 3. HPLC separation of HisrCssII and reduced HisrCssII.

The product obtained from the affinity column was directly loaded into the $\mathrm{C}_{18}$ reverse column (approx. $1 \mathrm{mg}$ of protein). The components labeled 1 to 10 had the molecular mass expected for the recombinant HisrCssII peptide of 9,392.6 Da (label A). Only the fractions 4 to 6 contained lethal material to mice at $20 \mu \mathrm{g} / 20 \mathrm{~g}$ of mice. The pooled multiple oxidized fractions $(0.5 \mathrm{mg}$ of protein, label A) were reduced with DTT, and loaded into the same HPLC. Superimposed to this chromatogram is the profile of the reduced HisrCssII (label B).

Fig. 4. HPLC separation of a properly folded HisrCssII and rCssII.

A). The in vitro oxidized peptide (100 $\mu \mathrm{g}$ protein, label A) was separated using an analytic $\mathrm{C}_{18}$ column. Superimposed in this chromatogram is a profile of the previously reduced HisrCssII (300 $\mu$ g protein, label B). B). The product of hydrolysis with protease Factor Xa was separated using an analytic $\mathrm{C}_{18}$ reverse-phase column. The HPLC trace corresponds to the digested material, showing the elution time of HisrCssII and rCssII, 
respectively.

Fig. 5. Circular dichroism of native (nCssII) and recombinants CssII.

The secondary structure fractions of the CD spectra according to Andrade et al. [17] were; $\alpha$-helix $0.14,0.14,0.15 ; \beta$-sheet $0.32,0.33,0.33$; and random coil $0.54,0.53,0.52$ for $\mathrm{rCss} 2$, nCss2, and $\mathrm{HisrCss} 2$, respectively.

Fig. 6. Electrophysiological experiments in voltage-gated sodium channels and competitive inhibition of binding of ${ }^{125}$ I-CssII by nCssII and rCssII.

A). Normalized conductance obtained, before and after application of $560 \mathrm{nM}$ nCssII, from experiments done on cells stably expressing the channel $\mathrm{Na}_{\mathrm{v}} 1.6$. Data are shown in control (closed squares) and during toxin application without prepulse (closed circles) or with prepulse (open triangles). Lines are best fit with sums of two Boltzmann relationships (of different amplitudes) representing channels bounded (toxin+pulse, half activation at $-56 \pm 1.5 \mathrm{mV}, \mathrm{n}=4$ ) or unbounded (control, half activation at $-16 \pm 1.2 \mathrm{mV}$, $\mathrm{n}=4$ ) by the peptide. Insets show superimposed traces, in an exemplary cell, at -50 and $10 \mathrm{mV}$ : upper left, control; upper right, toxin and no pulse; lower left, toxin and pulse. Notice that the toxin without pulse produced only a blocking effect and no left-shift. B). Normalized conductance obtained, before and after application of $560 \mathrm{nM}$ rCssII recombinant, from experiments similar to those shown in A and illustrated with the same symbols. Data are shown in control and during toxin application without or without prepulse. Lines are best fit with sums of two Boltzmann relationships representing channels bounded (toxin+pulse, half activation at $-72 \pm 8.5 \mathrm{mV}, \mathrm{n}=3$ ) or unbounded (control, half activation at $-23 \pm 2.2 \mathrm{mV}, \mathrm{n}=3$ ) by the peptide. Insets show superimposed traces, in an exemplary cell, at -50 and $-10 \mathrm{mV}$ : upper left, control; upper right, toxin and no pulse; lower left, toxin and pulse. Notice that the toxin rCssII produced effects very similar to those shown in A for toxin nCssII.

C). The amount of ${ }^{125}$ I-CssII bound is expressed as the percentage of the maximal specific binding without additional toxin. Non-specific binding, determined in the presence of $70 \mathrm{nM}$ CssII, was subtracted from all data points. The determined $\mathrm{IC}_{50}$ values were; nCssII: $0.10 \pm 0.03 \mathrm{nM}$ and rCssII: $1.54 \pm 0.03 \mathrm{nM}$. 
A

1 atAAAGAGGGCTATTTGGTAGCAAGAGCACAGGCTGCAAATACGAATGCCTTAAATTGG 60

$\begin{array}{lllllllllllllllllllllll}1 & K & \text { E } & G & Y & \text { L } & V & S & K & S & T & G & C & K & Y & E & C & \text { L } & K & \text { I } & G & & 20\end{array}$ $70 \quad 90 \quad 110$

61 GAGATAACGATTATTGCTTAGGGGAATGCAAACAGCAGTACGGAAAAAGCAGTGGCGGCT 120

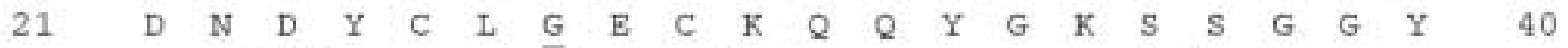
130 - 150 170

121 ATTGCTACGCTTTTGCGTGCTGGTGCACACACTTGTACGAACAAGCAGTGGTTTGGCCCC 180

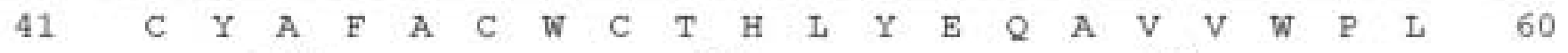
190
210
230

181 TTCCTAATAAAACATGCAACGGA.AATAAtggcaacgactttttattgtccactaacaga 240

$\begin{array}{llllllllllll}61 & \text { P } & \text { N } & \text { K } & \text { T } & \text { C } & \text { N } & \text { G } & \text { K } & + & 69\end{array}$ $250 \quad 270$

241 aatgttgtaacgettttaattcaattaatgaataaatgttataccttcagtaaaa 300 310

301 aaaaaaaaaaaaaaaaaaaaa 325

B

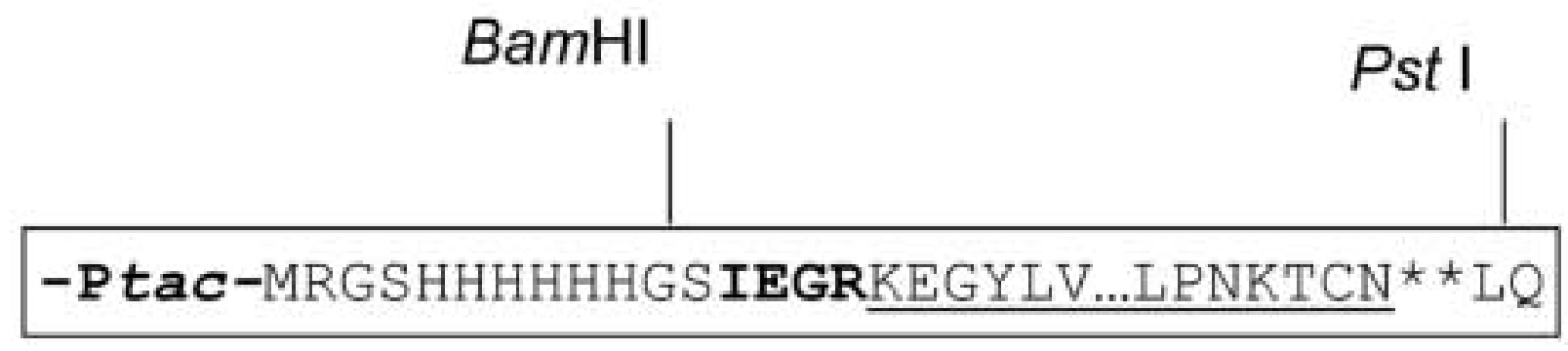

C

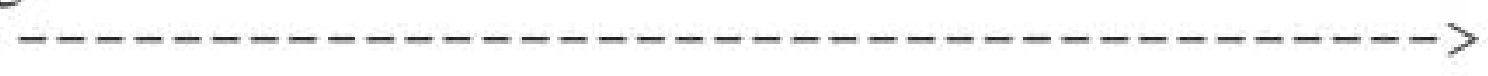

$\begin{array}{llllllllllllll}\mathrm{G} & \mathrm{S} & \mathrm{E} & \mathrm{G} & \mathrm{R} & \mathrm{K} & \mathrm{E} & \mathrm{G} & \mathrm{Y} & \mathrm{L} & \mathrm{V}\end{array}$

$5^{\circ}$ GGATCCATCGAGGGAAGGAAAGAGGGCTATCTGGTA A 3 * BamHI

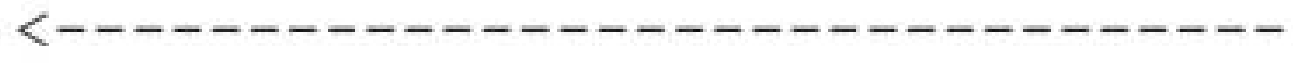

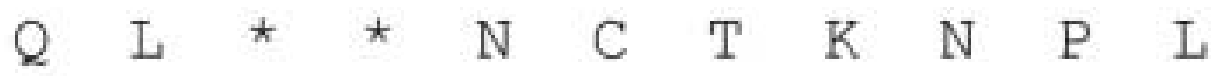

$5^{\circ}$ CTGCAGTTACTAGTTGCATGTTTTATTAGGAAG G 3 ' Pst I 
Figure 2
Click here to download high resolution image

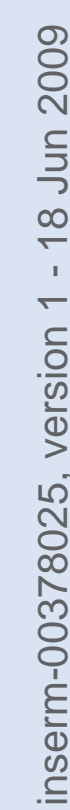
$\mathrm{KDa} 1$
2
3
4
5

175

47.5

32.5

25

16.5

6.5

HisrCssII $\longrightarrow$ 


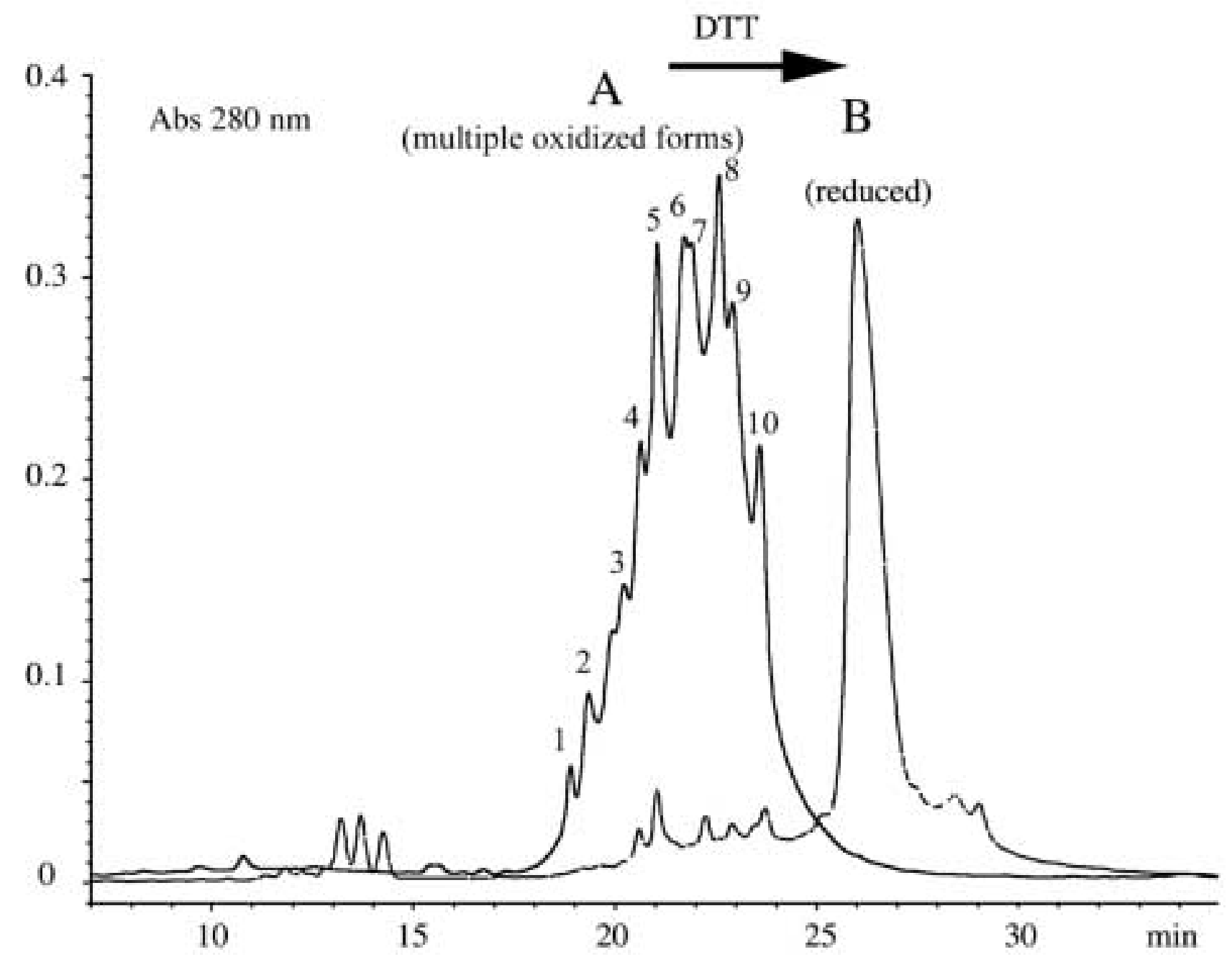


A

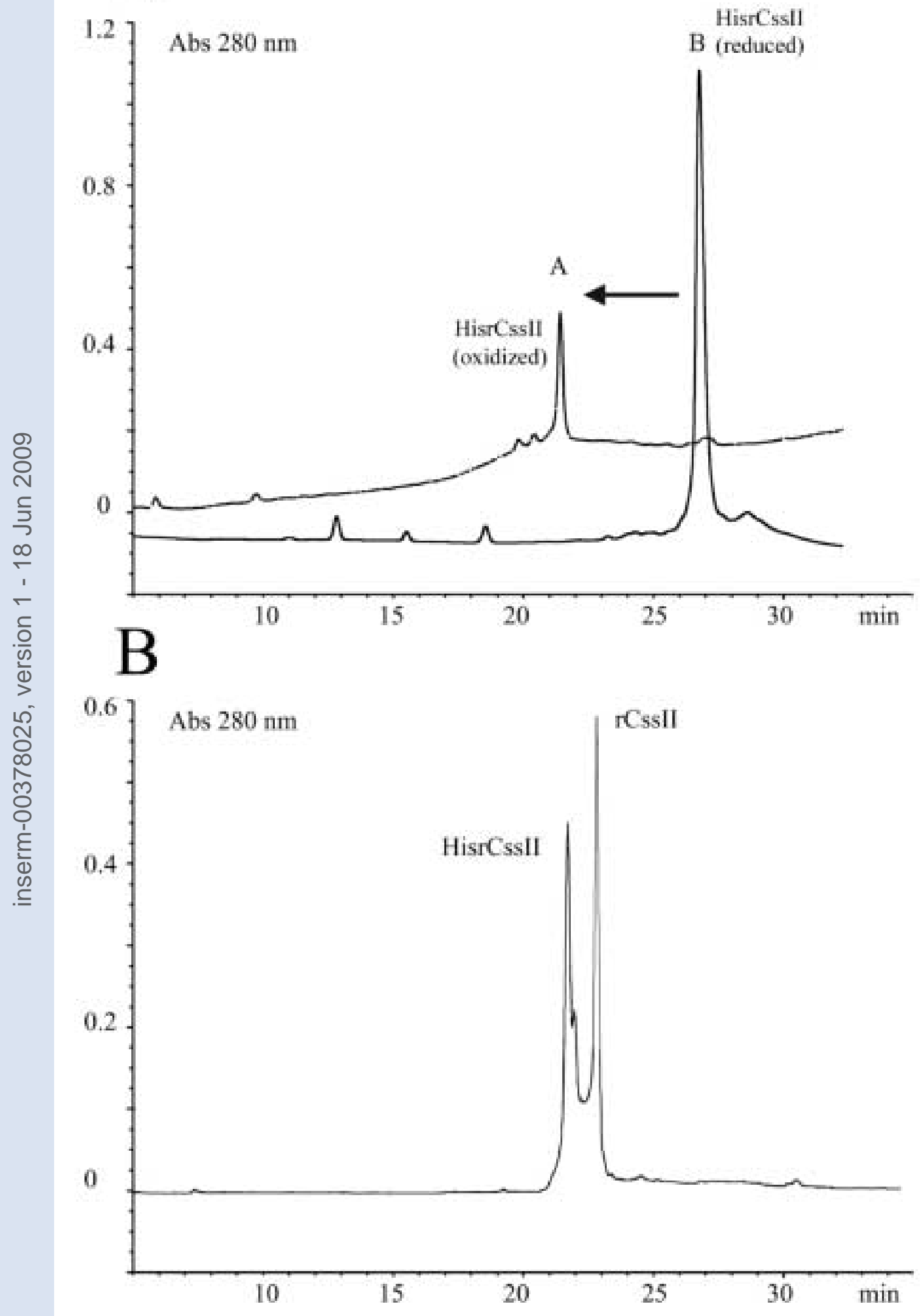




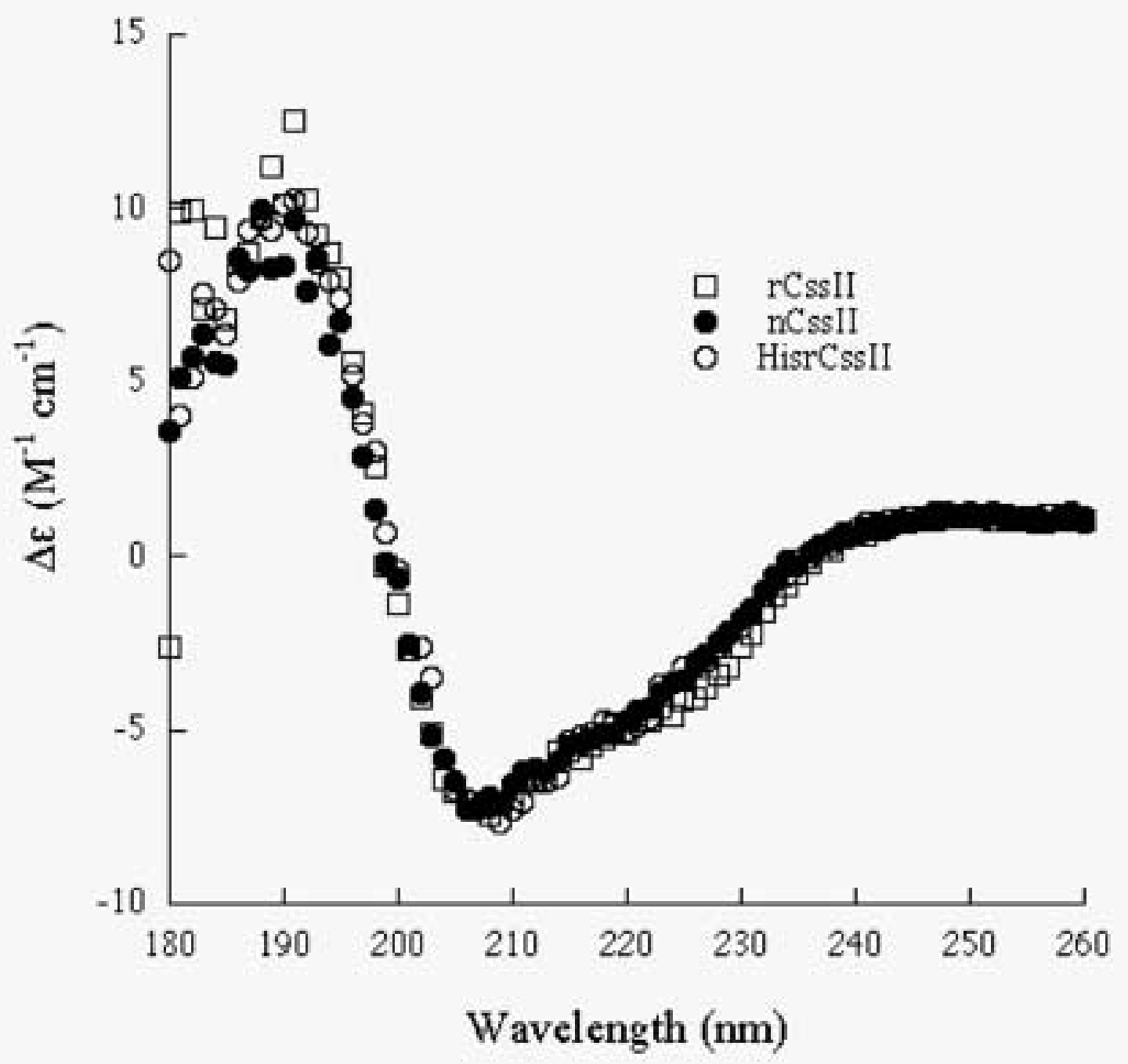


A

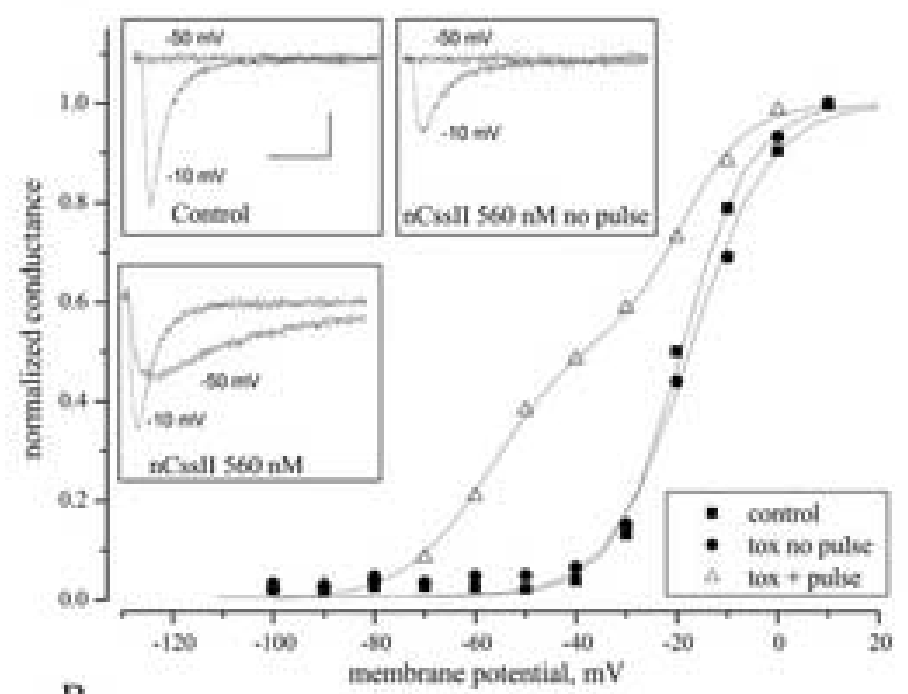

B

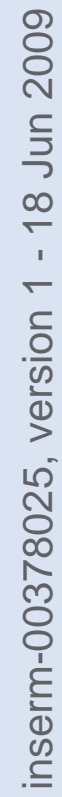

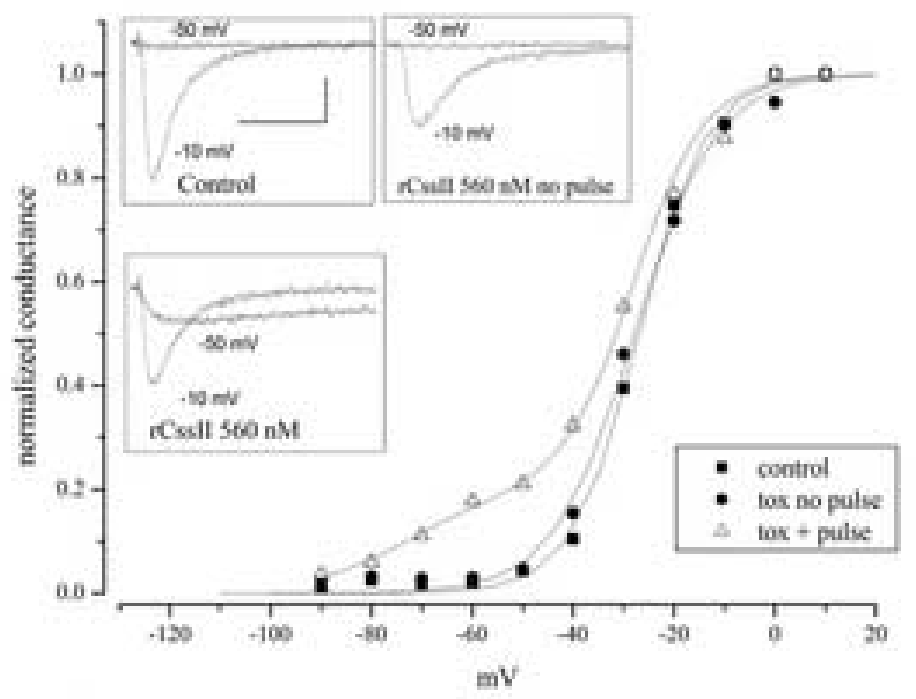

C

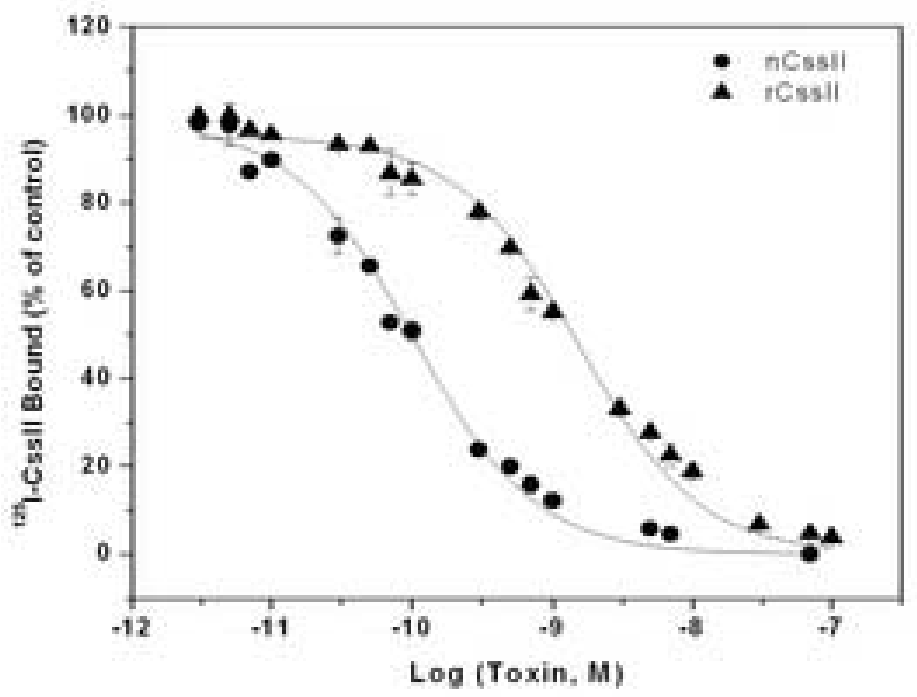

